\title{
A study of waveguide reactance element designs for introductory microwave experiments
}

\author{
Yusuke Kusama ${ }^{1 a)}$, Robert Weston Johnston ${ }^{1}$, \\ and Osamu Hashimoto ${ }^{2}$ \\ ${ }^{1}$ National Institute of Technology, Kagawa College, \\ 551 Kouda, Takuma-cho, Mitoyo, Kagawa 769-1192, Japan \\ 2 Aoyama Gakuin University, \\ 5-10-1 Fuchinobe, Chuo-ku, Sagamihara, Kanagawa 252-5258, Japan \\ a)kusama@cn.kagawa-nct.ac.jp
}

\begin{abstract}
The use of a triple combination of theory, simulation, and lab exercise is most commonly used to promote better student understanding of microwave fundamentals. As part of the RF engineers training education program, an advanced experiments program has been developed in which students can experience successive trial and error procedures including design, circuit fabrication, simulation, and measurement. As a result, it is expected that it be possible for other colleges of technology to use these methods to help students better understand the basis of practical microwave circuit design procedures. In this paper, the design theory and production evaluation process are focused on waveguide inductive and capacitive window.
\end{abstract}

Keywords: microwave circuit, waveguide, technical education, school education

Classification: Microwave and millimeter-wave devices, circuits, and modules

\section{References}

[1] Y. Takeda: A Radio Wave and Frequency Textbook (Impress, 2005) 54.

[2] S. Shiga: "The request from a microwave device company to institutions of a higher education," Electronics 1, Proc. of Institute of Electronics, Information, and Communication Engineers Japan, 2008 (2008) S29.

[3] S. Kawasaki: "Education in a university for microwave solid-state circuits: Class and laboratory for the circuit design using a simulator," Proc. of Institute of Electronics, Information, and Communication Engineers Japan (2002) 344.

[4] M. Vindevoghel, et al.: "Multimedia self-training product for microwave learning," Conf. Proc. EuMC, 20th, 1 (1990) 729 (DOI: 10.1109/EUMA.1990. 336129).

[5] R. E. Mc Intosh, et al.: "Microwave educational program: An industryuniversity alliance," IEEE Trans. Educ. 26 (1983) 143 (DOI: 10.1109/TE.1983. 4321632). 
[6] H. C. C. Fernandes, et al:: "PACMO: A comprehensive CAD package for microwave devices," IEEE Trans. Educ. 26 (1983) 162 (DOI: 10.1109/TE. 1983.4321635).

[7] D. M. Pozar: "A modern course in microwave engineering," IEEE Trans. Educ. 33 (1990) 129 (DOI: 10.1109/13.53636).

[8] W. Menzel: "Microwave education supported by animations of wave propagation effects," IEEE Trans. Microw. Theory Techn. 51 (2003) 1312 (DOI: 10.1109/TMTT.2003.809177).

[9] H. Kazuhiko: "Advanced seminar using microwave CAD - A new trend for seminars in universities for professional," The University of ElectroCommunications Bulletin 16 (2004) 169.

[10] I-Laboratory: "High Frequency Circuit Design Technology in GHz Era," http:// www1.sphere.ne.jp/i-lab/.

[11] Keysight Technologies: "High-frequency Measurement Engineering on Microwave Technology," Special lecture for doctoral course in graduate school of Tohoku University, http://www.keysight.com/.

[12] Y. Kusama and O. Hashimoto: "A study on development of experimental student program for RF engineer training-High-frequency impedance measurement with waveguide standing wave method-, "Technical Report of IEICE, ET2014-35 (2014) 35.

[13] Y. Kusama and M. Satoh: "Fabrication and characteristics measurement of a waveguide reactance element - For experimental graduate student program in microwave engineering_-, JSEE 62nd Annual Convention, 3F07 (2014) 538.

[14] Y. Konishi: High Frequency and Microwave Circuit_Basis and Design(Keilabo Publication, 2003) 277.

[15] S. Kawasaki and A. Nakatani: CAD Deign I of Microwave Planar Circuit_Passive Circuit_- (Realize Inc., 1996) 77.

[16] D. M. Pozar: Microwave Engineering (Wiley, 2011) 4th ed. 203.

[17] K. Hongo: Basis of The Electromagnetic Field and The Calculation Method (Shinzansha Scitech, 1993) 84.

[18] Uher, et al:: Waveguide Components for Antenna Feed Systems: Theory and CAD (Artech House, 1993) 9.

[19] Z. Kyo: CAD Programs for Microwave Circuit Engineers and Researchers (Mimatsu Data, 1998) 69.

[20] T. S. Chu and T. Itoh: "Generalized scattering matrix method for analysis of cascaded and offset microstrip step discontinuities," IEEE Trans. Microw. Theory Techn. 34 (1986) 280 (DOI: 10.1109/TMTT.1986.1133323).

\section{Introduction}

In the past decade, microwave based technology products have become something very familiar to the public through the widespread use of mobile communications, wireless LANs, GPS and automotive radar. From the perspective of the frequency allocation and reorganization policy presented by the Ministry of Internal Affairs and Communications (MIC) in Japan, the frequency of carrier waves is expected to go higher for better performance, more effective use and efficient information transmission [1]. Therefore, it is essential to cultivate deep theoretical knowledge and practical elemental technologies to cope with new microwave technology as a competent engineer. However, there has been a constant shortage of engineers with specialized knowledge and practical skills for microwave circuit and antenna 
design, so-called "RF Design" [2]. To survive in the field of RF design technology in the future, there is a necessary and urgent need for a large number of RF engineers who have excellent sense and creativity from the early stages. To train RF engineers who will be able to exert ingenious design perspectives, it is necessary for them to experience a large number of practical experiments in parallel with the theory behind supporting them with the help of simulators. Then both theoretical knowledge and practical techniques can be synergistically deepened [3].

Although it is fundamental to acquire theoretical knowledge during lecture, microwave engineering consists of versatile subjects such as materials, circuits, modules, antennae, systems, measurement, error analysis, theoretical mathematics, programming, and simulators. It is like a jigsaw puzzle made of many pieces. To support beginners, there is a need to repeat a systematic arrangement and maintenance of these puzzle pieces in accordance with the progress of the times and technology. While this requires a great deal of effort and cost, such as flip teaching or remote teaching delivery using the web as part of the active learning, it seems that there is still room for further improvement through the development of more information and communication technologies.

On the other hand, for measurement and evaluation, creativity and expert sense are cultivated gradually by repeated experiences of trial and error cycles unique to $\mathrm{RF}$ design, (design $\Rightarrow$ simulation $\Rightarrow$ production $\Rightarrow$ measurement evaluation $\Rightarrow$ problem discovery thinking and solving $\Rightarrow$ redesign). Generally, this type of cycle has only been experienced by a small portion of the technicians at many companies. It is the objective of the present study to introduce and expand a practical experiments course that can be experienced by students early on in higher educational institutions, including the National Institute of Technology. There are many publications about at this type of microwave education programs for reference in foreign countries. This includes the use of computer aided design (CAD), computer aided engineering (CAE), packaged integrated video materials and simulators, theoretical calculations, design exercises, measurement training, and introduction of microwave education courses in many universities $[4,5,6,7,8]$. In Japan, several examples of specialized education programs for graduate students or workers have been reported $[9,10,11]$.

In the microwave experiments course for 5 th grade students in the department of communication network engineering at NIT, Kagawa College, a practical experiment unit combining measurement, theoretical calculation, and simulation was introduced in order for students to perform derivation of high frequency load impedance using waveguide standing-wave pattern measurement [12]. Although in the previous experiment, three different load conditions (short, open, and load) that make up a commonly used calibration standard are examined, inductance or capacitance load which has important role in understanding the Smith chart have not been considered. So in order to newly introduce such reactance elements, we have reported on the evaluation results of the simplified samples fabricated as a preliminary test [13]. In this study, several new waveguide reactance elements are remanufactured to experimental standards to eliminate the influence of manufacturing error and were investigated. Good agreement was obtained compared with exact convention solution method. 


\section{Calculation theory}

\subsection{Equivalent circuit analysis}

Most familiar waveguide $L, C$ elements can be expressed by changing the length of the short ended waveguide with shunt connection and it is obtained apparently reactance shift. Another method is expressed by inserting the electric wall in the waveguide cross section. Although, the latter is somewhat cumbersome method compared to the former, it is an effective approach to miniaturization of the circuit. These are commonly referred to as inductive window or capacitive windows, relation between the electric wall size and the shunt equivalent circuit element values are obtained [14]. For example, it is possible to make a window of width $d$ in the waveguide section by inserting the metal wall of width $a-d$ shown in the upper part in Fig. 1. Where a is a waveguide width, $\mathrm{b}$ is the waveguide height. The $z$-axis is the propagation direction. Since the magnetic field is generated in the $x z$ plane to avoid the inserted metal wall, the magnetic field is generated around the metal wall. In this case the inductive reactance is obtained on the metal wall by the current flow in the y-axis direction. The normalized susceptance corresponding to the upper part of the inductive window L1 and L2 in Fig. 1 are shown in Equation (1) and (2). Here $Y_{0}$ is characteristic admittance of the waveguide, $B$ is susceptance of inductive window, $\lambda_{g}$ is the guided wavelength of the waveguide $\mathrm{TE}_{10}$ mode. The corresponding equation for structure L3 is omitted.

$$
\begin{gathered}
\frac{B}{Y_{0}}=-\frac{\lambda_{g}}{a}\left\{1+\operatorname{cosec}^{2}\left(\frac{\pi d}{2 a}\right)\right\} \cot ^{2}\left(\frac{\pi d}{2 a}\right) \\
\frac{B}{Y_{0}}=-\frac{\lambda_{g}}{a} \cot ^{2}\left(\frac{\pi d}{2 a}\right)
\end{gathered}
$$

Similarly, it is possible to make the window of the vertical width $d$ in the waveguide section by inserting the metal wall of width b-d shown in the bottom in Fig. 1. Thus interval of waveguide E-plane is narrowed by inserting a metal wall, the electric field is concentrated in the $y z$ plane. In this case the capacitive reactance is obtained on the metal wall gap by the charge storage in the $y$-axis direction. The normalized susceptance corresponding to the lower part of the capacitive windows $C 1$ and $C 2$ in Fig. 1 are shown in Equation (3) and (4). The corresponding equation for structure $C 3$ is omitted.

$$
\begin{aligned}
& \frac{B}{Y_{0}}=\frac{8 b}{\lambda_{g}} \ln \left\{\operatorname{cosec}\left(\frac{\pi d}{2 b}\right)\right\} \\
& \frac{B}{Y_{0}}=\frac{4 b}{\lambda_{g}} \ln \left\{\operatorname{cosec}\left(\frac{\pi d}{2 b}\right)\right\}
\end{aligned}
$$

As from the equation (1) to (4), it is very useful the value of the element is given as a function in advance because beginners can derive calculation value easily in Excel though derivation of these equations are even as advanced content. When measuring such a device under test (DUT) elements in waveguide systems, it is considered an equivalent circuit as shown in Fig. 2. The normalized input impedance zin shown from Port1 is the parallel connection of $B$ and $Y_{0}$. It is given by

$$
z_{\text {in }}=\frac{Z_{\text {in }}}{Z_{0}}=\frac{1}{y_{\text {in }}}=\frac{1}{1+j b}=\frac{1}{1+b^{2}}+j \frac{-b}{1+b^{2}}
$$


Here, $Y_{0}$ is the characteristic admittance of waveguide $\mathrm{TE}_{10}$ mode, $b=B / Y_{0}$ is a normalized susceptance of the DUT. Therefore, reflection coefficient $\Gamma$ from Port1 is given by

$$
\Gamma=\frac{Z_{\text {in }}-Z_{0}}{Z_{\text {in }}+Z_{0}}=\frac{z_{\text {in }}-1}{z_{\text {in }}+1}=\frac{(\alpha+j \beta)-1}{(\alpha+j \beta)+1}
$$

And the real part and imaginary part of the reflection coefficient is given by the following equations.

$$
\begin{aligned}
& \operatorname{Re}[\Gamma]=\frac{\alpha^{2}+\beta^{2}-1}{(\alpha+1)^{2}+\beta^{2}}=\operatorname{Re}\left[S_{11}\right] \\
& \operatorname{Im}[\Gamma]=\frac{2 \beta}{(\alpha+1)^{2}+\beta^{2}}=\operatorname{Im}\left[S_{11}\right]
\end{aligned}
$$

Here, the $\alpha$ and $\beta$ in equation (6) to (8) represents the real part and the imaginary part of the normalized impedance of equation (5), it is the respective next value.

$$
\alpha=\frac{1}{1+b^{2}}, \quad \beta=\frac{-b}{1+b^{2}}
$$
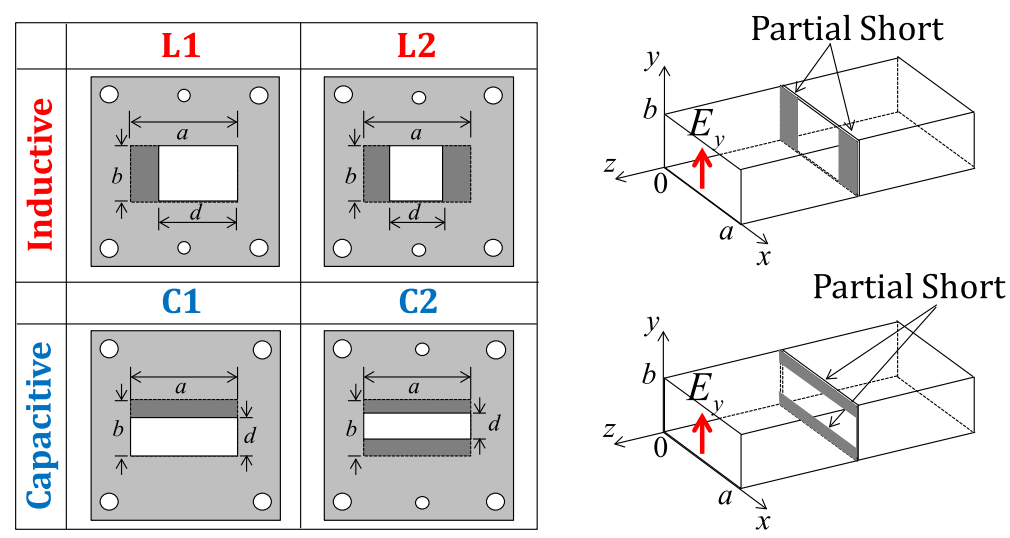

Fig. 1. Examples of inductive window and the capacitive window. $a \times b$ is the original waveguide cross section, and $d$ represents the window size. $L 1 ; C 1$ are the window structure of one side only, $L 2 ; C 2$ are center window structure.

\subsection{Mode matching method}

Equivalent circuit analysis of the previous section 2.1 is a simple expression that focuses on the $\mathrm{TE}_{10}$ dominant mode. So it is necessary to take into account the higher-order modes that occur in the vicinity of the window. To cope with these problems, an electromagnetic field simulator capable of full-wave analysis or the mode matching method to connect the mode series sum expressed in the form of eigenfunction expansion at the boundary surfaces are known $[15,16]$. Analysis of the inductive window is reduced to H-plane discontinuity problem, although the analysis of the capacitive window is reduced to E-plane discontinuity problems. It will be described here only the latter. Consider no discontinuity in the waveguide $\mathrm{x}$ direction as shown in Fig. 3. When the driven mode can be assumed to $\mathrm{TE}_{m 0}$, the electric field component $E_{x}$ does not occur structurally. Thus, it can be developed with $\mathrm{TE}_{x}\left(\mathrm{LSE}_{x}\right.$ mode) considering the five electromagnetic field components [17]. By using the following electric vector potential $F$ 

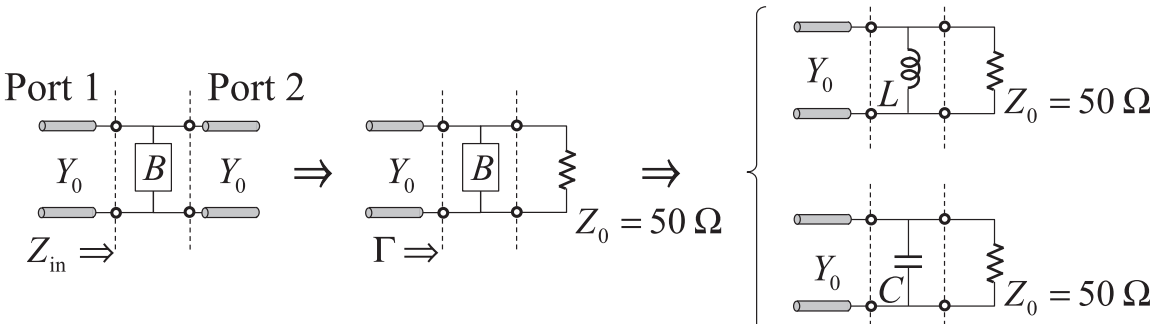

Fig. 2. Equivalent circuits for the DUT measurement.

$$
\begin{aligned}
& \mathbf{F}^{+}=\hat{x} F_{x}{ }^{+}=\hat{x} F_{m n} \sin \left(\frac{m \pi}{a} x\right) \cos \left(\frac{n \pi}{b} y\right) e^{-j k_{z} z} \\
& \mathbf{F}^{-}=\hat{x} F_{x}^{-}=\hat{x} B_{m n} \sin \left(\frac{m \pi}{a} x\right) \cos \left(\frac{n \pi}{b} y\right) e^{+j k_{z} z}
\end{aligned}
$$

The electromagnetic field of the $\mathrm{TE}_{x}$ mode can be expressed by the following equation.

$$
\begin{aligned}
& E_{x}=0, \quad H_{x}=-j \frac{1}{\omega \mu \sigma}\left(\frac{\partial^{2}}{\partial x^{2}}+\omega^{2} \mu \varepsilon\right) F_{x} \\
& E_{y}=-\frac{1}{\varepsilon} \frac{\partial F_{x}}{\partial z}, \quad H_{y}=-j \frac{1}{\omega \mu \varepsilon} \frac{\partial^{2} F_{x}}{\partial x \partial y} \\
& E_{z}=\frac{1}{\varepsilon} \frac{\partial F_{x}}{\partial y}, \quad H_{z}=-j \frac{1}{\omega \mu \varepsilon} \frac{\partial^{2} F_{x}}{\partial x \partial z}
\end{aligned}
$$

For example, in order to apply the boundary condition at the discontinuity $z=0$, the $n$-th mode of the transverse electromagnetic field set of $E_{y}, H_{x}$ are expressed by

$$
\begin{aligned}
& E_{y}=G_{n} \sin \left(k_{x} x\right) \cos \left(k_{y} y\right)\left(F_{n} e^{-j k_{z} z}+B_{n} e^{+j k_{z} z}\right) \\
& H_{x}=-G_{n} Y_{n} \sin \left(k_{x} x\right) \cos \left(k_{y} y\right)\left(F_{n} e^{-j k_{z} z}-B_{n} e^{+j k_{z} z}\right)
\end{aligned}
$$

Here, fixed $m=1$ for the $\mathrm{TE}_{10}$ mode input. The wave number $k_{z}$, cutoff wave number $k_{c}$, and admittance $Y_{n}$ is given by equation (17) to (19).

$$
\begin{aligned}
& k_{z}= \begin{cases}\sqrt{k_{0}^{2}-k_{c}^{2}} & \left(k_{0}>k_{c}\right) \\
-j \sqrt{k_{c}^{2}-k_{0}^{2}} & \left(k_{0}<k_{c}\right)\end{cases} \\
& k_{c}=\sqrt{k_{x}^{2}+k_{y}^{2}}=\sqrt{\left(\frac{\pi}{a}\right)^{2}+\left(\frac{n \pi}{b}\right)^{2}} \\
& Y_{n}=\frac{k_{0}^{2}-k_{x}^{2}}{\omega \mu k_{z}}
\end{aligned}
$$

Also, $G_{n}$ of the equation (20) is a normalized amplitude when transmitted power of the propagation mode in $z$-direction is $1 \mathrm{~W}$ and also, transmitted power of the nonpropagation mode is $j 1 \mathrm{~W}$. Here, $\varepsilon_{n}$ is a symbol shown in equation (21).

$$
\begin{aligned}
& G_{n}= \begin{cases}\sqrt{\frac{\varepsilon_{n}}{a b} \frac{1}{Y_{n}}} \quad\left(k_{0}>k_{c}\right) \\
\sqrt{\frac{\varepsilon_{n}}{a b} \frac{-j}{Y_{n}}} \quad\left(k_{0}<k_{c}\right)\end{cases} \\
& \varepsilon_{n}= \begin{cases}1 & (n=0) \\
2 & (n \geq 1)\end{cases}
\end{aligned}
$$


Then, the boundary conditions of the following equation (22) (23) are applied at the $z=0$ plane. Although the right-hand side bottom in equation (23) correspond to the wall current, it may remain unknown.

$$
\begin{gathered}
\sum_{n=0}^{N} G_{n}^{I} \cos \left(k_{y}^{I} y\right)\left(F_{n}^{I}+B_{n}^{I}\right)= \begin{cases}\sum_{q=0}^{Q} G_{q}^{I I} \cos \left(k_{y}^{I I} y\right)\left(F_{q}^{I I}+B_{q}^{I I}\right) & 0 \leq y \leq b_{2} \\
0 & b_{2} \leq y \leq b_{1}\end{cases} \\
\sum_{n=0}^{N}-G_{n}^{I} Y_{n}^{I} \cos \left(k_{y}^{I} y\right)\left(F_{n}^{I}-B_{n}^{I}\right)= \begin{cases}\sum_{q=0}^{Q}-G_{q}^{I I} Y_{q}^{I I} \cos \left(k_{y}^{I I} y\right)\left(F_{q}^{I I}-B_{q}^{I I}\right) & 0 \leq y \leq b_{2} \\
? & b_{2} \leq y \leq b_{1}\end{cases}
\end{gathered}
$$

Furthermore, in order to use the mode orthogonality, equation (22) is multiplied by $\cos \left(k^{1}{ }_{y}\right)$ and integrated over the range from 0 to $b_{1}$ and also equation (23) is multiplied by $\cos \left(k^{I I}\right)$ and integrated over the range from 0 to $b_{2}$ each. Finally, they are summarized in the simultaneous equations shown in equation (24) [18]. In this study, the highest order mode number of both regions I and II are determined $N=Q=100$ on confirming the modal convergence. Here, $L_{E}, L_{H}$ are expressed in the form of equation (25) and (26). Although, these calculations include integral, the integral calculation is avoidable by using the addition theorem [19]. In the case of the capacitive window, a structure obtained by mirror-reversed image of Fig. 3 needs to be cascaded to the right through the thickness of the sample [20].

$$
\begin{aligned}
& \left\{\begin{array}{l}
F_{n}^{I}+B_{n}^{I}=\sum_{q=0}^{Q}\left[L_{E}\right]_{n, q}\left[F_{q}^{I I}+B_{q}^{I I}\right] \\
\sum_{n=0}^{N}\left[L_{H}\right]_{q, n}\left[F_{n}^{I}-B_{n}^{I}\right]=F_{q}^{I I}-B_{q}^{I I}
\end{array}\right. \\
& {\left[L_{E}\right]_{n, q}=\frac{\varepsilon_{n} G_{q}^{I I}}{b_{1} G_{n}^{I}} \int_{0}^{b_{2}} \cos \left(k_{y}^{I I} y\right) \cos \left(k_{y}^{I} y\right) d y} \\
& {\left[L_{H}\right]_{q, n}=\frac{\varepsilon_{q} G_{n}^{I} Y_{n}^{I}}{b_{2} G_{q}^{I I} Y_{q}^{I I}} \int_{0}^{b_{2}} \cos \left(k_{y}^{I} y\right) \cos \left(k_{y}^{I I} y\right) d y}
\end{aligned}
$$

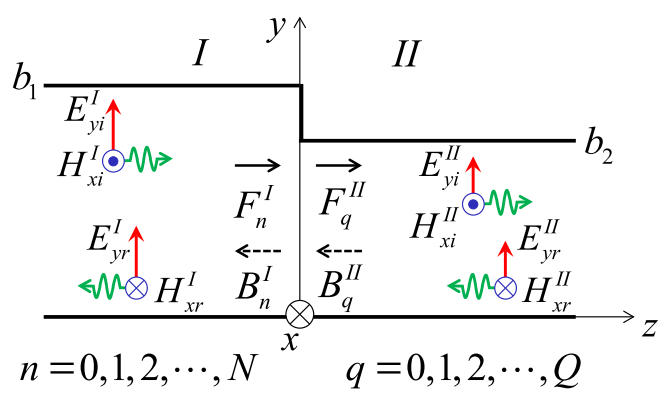

Fig. 3. Modal analysis model of $\mathrm{E}$ plane discontinuity for $\mathrm{TE}_{x}\left(\mathrm{LSE}_{x}\right)$ mode.

$F^{I}{ }_{n}$ and $B^{I}{ }_{n}(n=0,1,2, \ldots, N)$ are the normalized amplitude of nth order incident wave and reflected wave in region I. $F^{I I}{ }_{q}$ and $B^{I I}{ }_{q}(q=0,1,2, \ldots, Q)$ are the normalized amplitude of nth order incident wave or reflected wave in region II. 


\section{Measurement sample}

The sample shapes of the inductive window are shown in Fig. 4 left. Inductive windows were manufactured two kinds. One is the case of inserting the step structure only in the one side of the waveguide H-plane and the other is a symmetrical step structure. The prepared window sizes are the following three types of $80 \%, 50 \%$ and $20 \%$ with respect to the original waveguide cross section. Also, the sample shapes of capacitive window are shown in Fig. 4 right. Capacitive windows were manufactured also two kinds. One is the case of inserting the step structure only in the top side of the waveguide E-plane and the other is a symmetrical step structure. The prepared window sizes are the following three types of $80 \%, 50 \%$ and $20 \%$ with respect to the original waveguide cross section. The X-band standard waveguide WR-90 flange was used with Ag plated phosphor bronze. The thickness of the sample is sufficiently thin with respect to the wavelength, and was $0.5 \mathrm{~mm}$ so as to maintain the strength required for the measurement operation. It is also possible to own process using a commercially available copper plate as long as the thickness of this level.
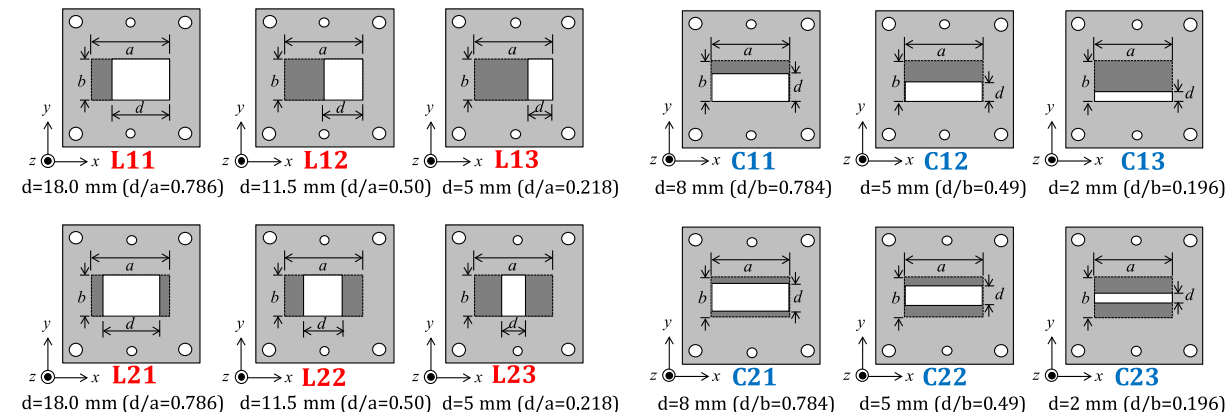

Fig. 4. Inductive window samples (left). From top left L11, L12, L13, and from the lower left L21, L22, L23. Capacitive window samples (right). From top left $\mathrm{C} 11, \mathrm{C} 12, \mathrm{C} 13$, and from the lower left $\mathrm{C} 21, \mathrm{C} 22, \mathrm{C} 23.0 .5 \mathrm{~mm}$ thickness phosphor bronze with WR-90 standard waveguide flange is used.

\section{Measurement system}

The measurement system is shown in Fig. 5. The tip of WR-90 coaxial to waveguide adapter is set to the calibration plane. The reflection characteristics $S_{11}$ were measured in the frequency range from 8.2 to $12.4 \mathrm{GHz}$ inserting the measurement sample into the calibration plane. The vector network analyzer (VNA) Agilent E8362C is used using the following conditions, IF bandwidth $1 \mathrm{kHz}$, the number of measurement points is 401 .

\section{Results}

Results of the inductive windows are shown in Fig. 6. The vertical axis is the reflection coefficient $[\mathrm{dB}]$ and the horizontal axis is the frequency $[\mathrm{GHz}]$. Three types of data indicate measured value (Meas.), equivalent circuit calculation (Circ.), and mode matching method (MM.). Focusing on L11 in Fig. 6 left, the cut-off frequency $6.5 \mathrm{GHz}$ of the input $\mathrm{TE}_{10}$ and the cut-off frequency $13 \mathrm{GHz}$ of the $\mathrm{TE}_{20}$ 

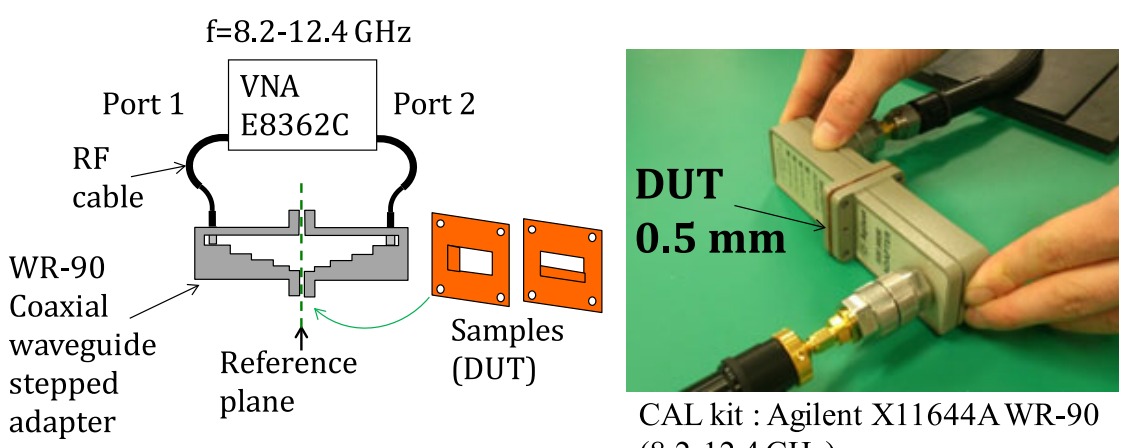

CAL kit : Agilent X11644A WR-90 $(8.2-12.4 \mathrm{GHz})$

Fig. 5. Schematic view of the measurement system. The calibration plane is set to the center of the WR-90 stepped coaxial to waveguide adaptor. The vector network analyzer (VNA) of Agilent E8362C is used for frequency range from 8.2 to $12.4 \mathrm{GHz}$, IF bandwidth $1 \mathrm{kHz}$, the number of measurement points is 401 .

can be confirmed by the mode matching method. Measured value and modematching method is in good agreement, but the calculation error becomes larger near the cut-off frequency of the $\mathrm{TE}_{20}$ mode by the equivalent circuit calculation. This trend is same with L12 which has relatively large reflection. Note that L13 is almost total reflection, it is difficult to distinct the difference between three data. On the contrary, looking at L21 in Fig. 6 right, it is not able to be confirmed $\mathrm{TE}_{20}$ cutoff frequency seen in Fig. 6 left. This is because the higher order mode of evennumber does not occur in a symmetrical structure. Although the mode matching method is in good agreement with the measured values in all case, the larger the error of the equivalent circuit calculation is if the reflection is small as in the case of L21. Moreover, in comparison to Fig. 6 left and right, it can be seen that the reflection coefficient varies by the relationship between the electromagnetic field distribution of the input mode and the window structure even though the window size is same.

Similarly, Results of the capacitive window are shown in Figs. 7. First, paying attention to the $\mathrm{C} 11$ in Fig. 7 left, the mode matching method and the equivalent circuit calculation are in good agreement. However, there is a significant difference between both calculation data and the measured value compared with the case of
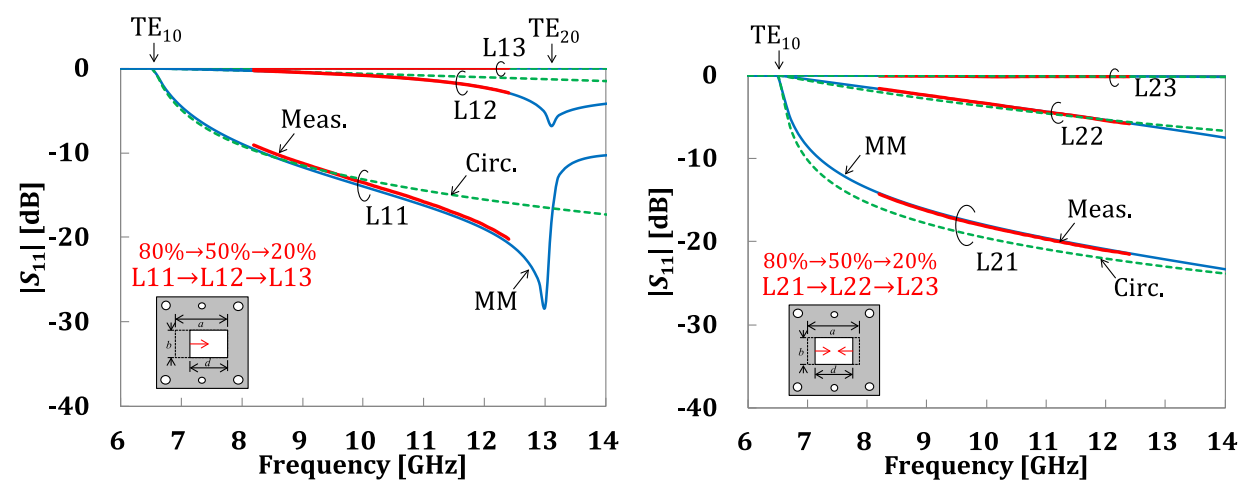

Fig. 6. Measurement result. Inductive window L11, L12, L13 (left). Inductive window L21, L22, L23 (right). 
the inductive window. In addition, in some cases in $\mathrm{C} 12$ and $\mathrm{C} 13$, the equivalent circuit calculation is close to the measured value than the exact mode matching method. It is considered that there is a reason that is still undissolved except for dimension errors. In addition, although C21, C22 and C23 in Fig. 7 right have same window size each with Fig. 7 left, it is equivalent to the half dimension of Fig. 7 left by the effect of the electric wall. Though the mode matching method and the measurement value are in relatively good agreement, it did not lead to an exact match such as the case of the inductive window.
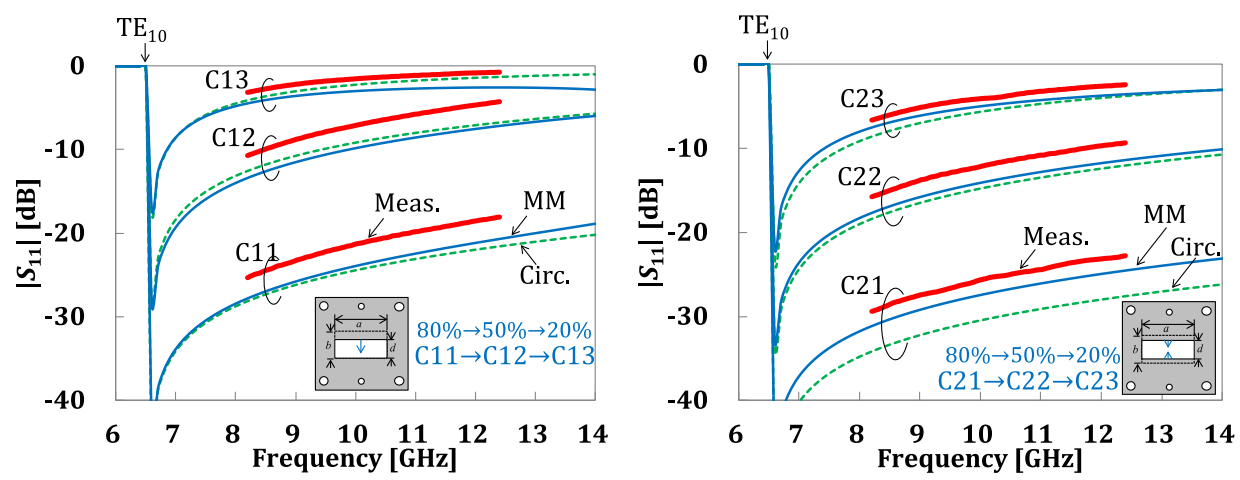

Fig. 7. Measurement result.

Capacitive window C11, C12, C13 (left). Capacitive window C21, C22, C23 (right).
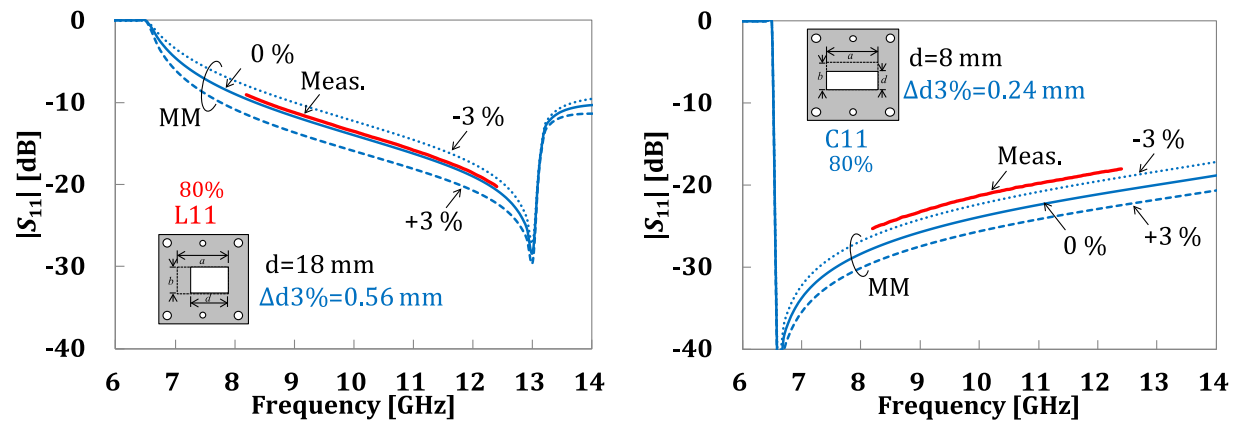

Fig. 8. Manufacturing error analysis. Inductive window L11 (left). Capacitive window C11 (right).

Fig. 8 shows the manufacturing error estimation. Two samples are investigated, one is the case of the inductive window L11, and the other is the case of the capacitive window $\mathrm{C} 11$. The measured valued is plotted from $8.2 \mathrm{GHz}$ to $12.4 \mathrm{GHz}$. And the manufacturing error of $+3 \%$ and $-3 \%$ are considered in the calculation. In the case of the L11, the $3 \%$ error corresponds to $0.56 \mathrm{~mm}$ on $\mathrm{H}$ plane. Also, in the case of the $\mathrm{C} 11$, the $3 \%$ error corresponds to $0.24 \mathrm{~mm}$ on $\mathrm{E}$ plane. It was found that the capacitive window is more sensitive in manufacturing error compared with the inductive window about two times in this case.

\section{Acknowledgments}

This research was subsidized by the scientific research fund 26750073, "Development of RF design engineer training program," young scientists (B). 